\title{
Considerations for an Earth Relay Satellite with RF and Optical Trunklines
}

\author{
David J. Israel ${ }^{1}$ \\ National Aeronautics and Space Administration Goddard Spaceflight Center, Greenbelt, MD, 20771
}

\begin{abstract}
Support for user platforms through the use of optical links to geosynchronous relay spacecraft are expected to be part of the future space communications architecture. The European Data Relay Satellite System (EDRS) has its first node, EDRS-A, in orbit. The EDRS architecture includes space-to-space optical links with a Ka-Band feeder link or trunkline. NASA's Laser Communications Relay Demonstration (LCRD) mission, originally baselined to support a space-to-space optical link relayed with an optical trunkline, has added an RF trunkline. The use of an RF trunkline avoids the outages suffered by an optical trunkline due to clouds, but an RF trunkline will be bandwidth limited. A space relay architecture with both $R F$ and optical trunklines could relay critical realtime data, while also providing a high data volume capacity. This paper considers the relay user scenarios that could be supported, and the implications to the space relay system and operations. System trades such as the amount of onboard processing and storage required, the use of link layer switching vs. network layer routing, and the use of Delay/Disruption Tolerant Networking (DTN) are discussed.
\end{abstract}

\section{Introduction}

NASA is working towards a next generation communications and navigation architecture that includes an optical relay capability [1]. Optical communication has been previously demonstrated for space applications and is in the early stages of deployment into operational systems. Optical communication (or lasercom for short) can provide much higher data rates for the available spectrum, reduced system size, weight, and power $(\mathrm{SWaP})$ requirements, reduced susceptibility to interference, and more secure connections. As lasercom technology continues to improve in performance, system availability, and costs, lasercom links to Earth will always face the inevitable - clouds happen. Periodically a cloud will pass between a ground station and a spacecraft, and the link will be dropped. For the direct to Earth (DTE) user, NASA's Lunar Laser Communications Demonstration (LLCD), which launched in September 2013 as a payload aboard the Lunar Atmosphere and Dust Environment Explorer (LADEE), proved the feasibility of laser communications reaching speeds of $622 \mathrm{Mbps}$ from lunar orbit. With developing technology, these rates are expected to increase. A low Earth orbiting satellite may achieve ultra-high DTE data rates in excess of 100 Gbps [2]. These DTE data rates, however, will only be able to transfer data when in view of a compatible ground station that is also not blocked by clouds. This results in limited opportunities that may not provide the data within the desired timeliness or latency requirement.

Space relay systems, such as NASA's Tracking Data and Relay Satellite System (TDRSS), with their increased coverage, have demonstrated that they can provide low latency RF services, and deliver high data volumes. For a space relay system providing optical link services, there is a fundamental challenge: how can the relay provide the maximum data capacity, while also providing the global coverage and availability expected from space relays?

The European Data Relay System (EDRS) will provide the full orbital coverage with reliable data delivery by using Ka-Band RF trunklines [3]. The first EDRS Satellite, EDRS A, was launched in January 2016, and

1 ESC Architect, Exploration \& Space Communications Projects, NASA GSFC/Code 450, Greenbelt, MD 20771, AIAA Senior Member 
started service in July 2016 [4]. The Ka-Band links have the necessary link margin to assure the delivery of data from the relay to Earth, once the laser communications link is received at the relay spacecraft. The primary limitation with this system design is that the relay data capacity will be limited by the spectrum constraints imposed on the RF links. These constraints will always be smaller in bandwidth than the potential data bandwidth lasercom can provide.

NASA's Laser Communications Relay Demonstration (LCRD) Project will expand on NASA's past successes in laser communications to provide extended operations experiments and demonstrations. LCRD will carry two optical communications terminals and associated electronics as a payload on a satellite in geosynchronous orbit to be launched in 2019. The two optical ground stations for LCRD will be located in Southern California and Hawaii. LCRD will provide at least two years of optical communications services in an operations environment, and is expected to provide bidirectional user data rates up to $1.244 \mathrm{Gbps}$.

LCRD's baseline design had only an optical trunkline with one of its two optical space terminals. Prior to the launch of an orbiting user, LCRD would demonstrate an optical to optical relay between its two ground stations with one station acting as the user and one as the receiving ground station [5]. This system design allowed the trunkline to carry the same bandwidth as the link between the user and the relay. This is ideal, until the inevitable cloud shows up. In this scenario, the optical trunkline would either need to wait for clouds to pass or switch to a different ground station that has a cloud free line of sight. Either way, a link outage occurs.

This paper will focus on the addition of an RF trunkline, and its benefits to the system. Consideration is also given to the relay user scenarios that should be supported, and the implications to the space relay system and operations. System trades such as the amount of onboard processing and storage required, the use of link layer switching vs. network layer routing, and the use of Delay/Disruption Tolerant Networking (DTN) are discussed.

\section{Adding a Radio Frequency Trunkline}

LCRD has recently added a high bandwidth RF trunkline to its design [Fig. 1]. This additional path now allows for some user data to be delivered while an optical outage occurs [Fig. 2]. An additional RF system aboard the relay does add additional cost and complexity. However, the added capability allows each trunkline link type, RF and optical, to complement the other. The optical link can deliver the data volume

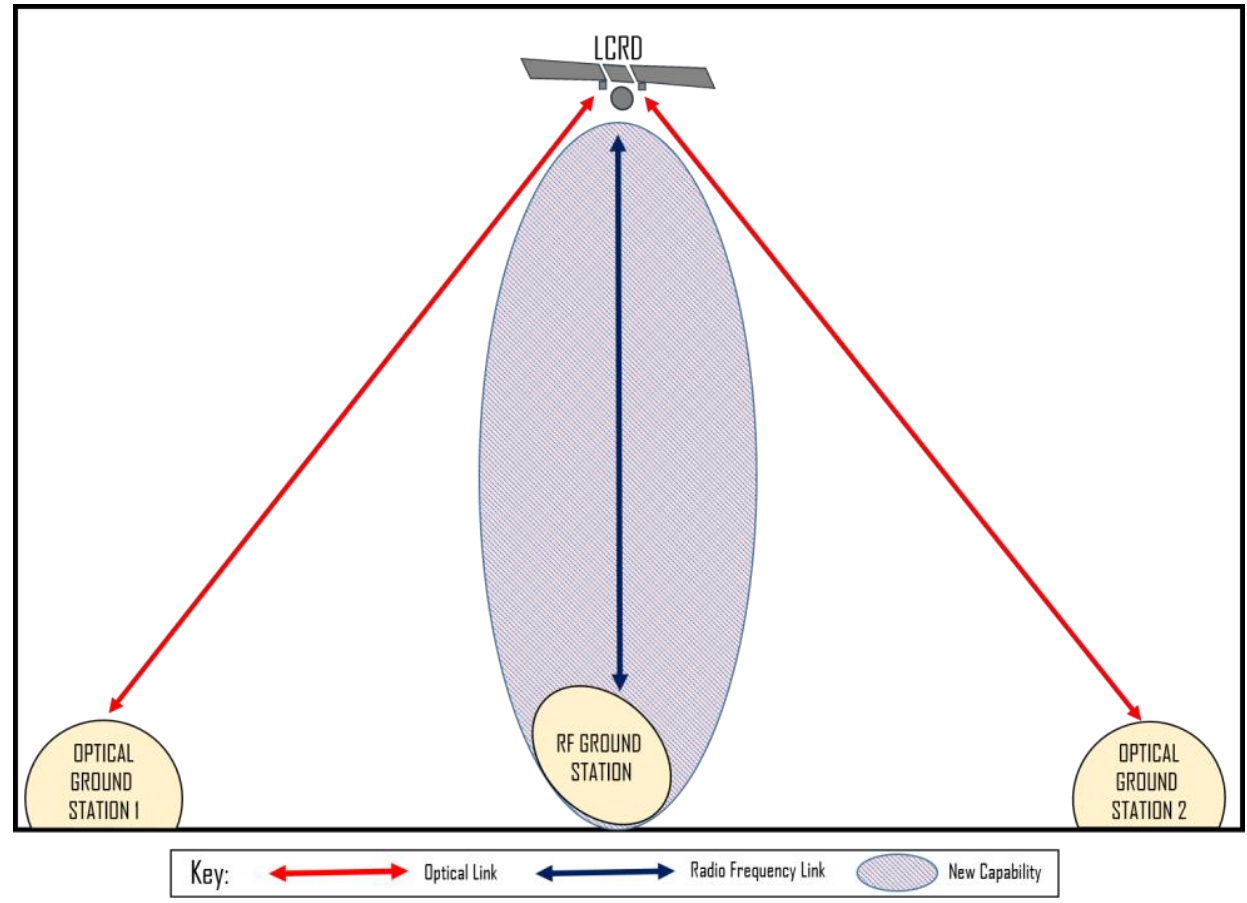

Figure 1. RF Trunkline Added to Original LCRD Design 
that is too much to fit within the RF spectrum and the RF link can deliver the data that cannot tolerate the outages that the optical link will suffer due to clouds.

The assumption is made that some user data can tolerate the outages, usually by accepting delayed data delivery due to required retransmissions. Most missions have data with realtime or very low latency delivery requirements (commanding, telemetry, science alerts, voice, video, etc.) and data that can be delayed as long as it is completely delivered (science instrument files, housekeeping files, software uploads, etc.) [6].

By adding the RF trunkline, this system provides a best of both worlds scenario to the user.

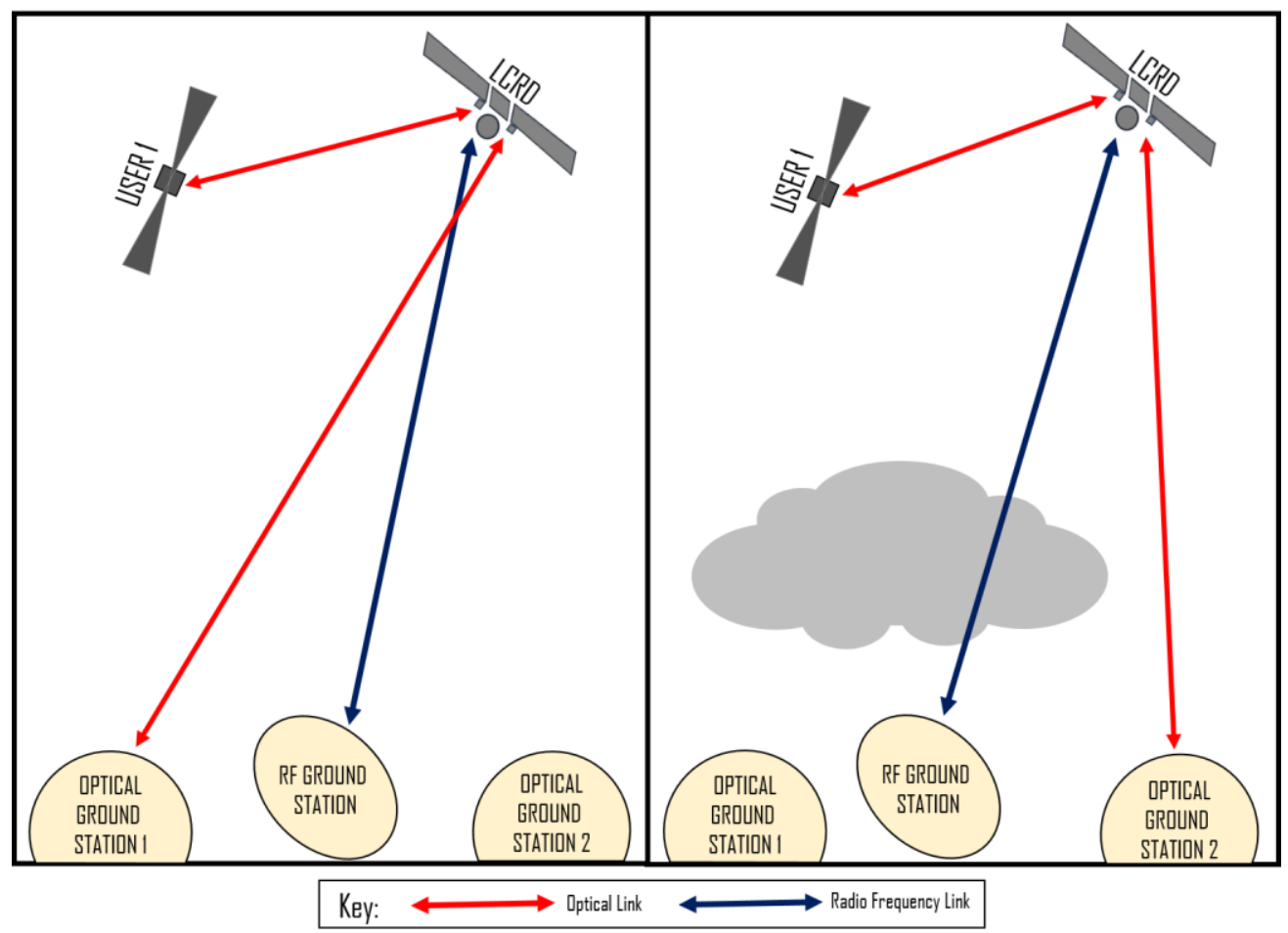

Fig. 2. RF Trunkline Enables Transmission of Critical Data during Cloud Cover

\section{A. Single Trunkline vs Dual Trunklines}

The design choice to include both an optical and a RF trunkline will lead to many new trades concerning their specifications and the overall operations concept. One trade is the determination of which data uses each trunkline. It is assumed that all RF and optical links are supported by modems in the flight payload, and, at minimum, the system is a demod/remod system. One option would be to select the trunkline for a relay path a priori, such that data for a certain user either all traverses the RF trunkline or all traverses the optical trunkline [Fig. 3a]. This simplifies the flight payload and relay operations greatly, since no onboard processing is required beyond basic modulation and demodulation. In the case of using the RF trunkline, if the user data rate fits within the available bandwidth, delivery would be guaranteed. If the user requirements could all be met within the possible latencies, then the optical trunkline would be capable of delivering a higher data volume.

The coarse assignment of the trunkline at the user level may simplify the relay payload requirements but leads to scheduling constraints and likely link utilization inefficiencies. If a portion of the RF or optical trunkline is allocated for use, an additional user service can only be added if it all fits into either trunkline. Switching the data paths at the frame level [Fig. 3b] would allow each trunkline to be better utilized by taking advantage of the earlier assumption that a single user would have both realtime and latency tolerant data. To operate this way, the user data would have to be transmitted in a defined common frame format, such that the relay's onboard processing could switch the frames between destinations as they are received. User systems would be required to not only frame their data, but to also mark each frame in a defined way to indicate 
priority or latency tolerance. Note that channel coding and decoding is not required onboard the relay if the frame headers themselves are not encoded. This option is the LCRD implementation.

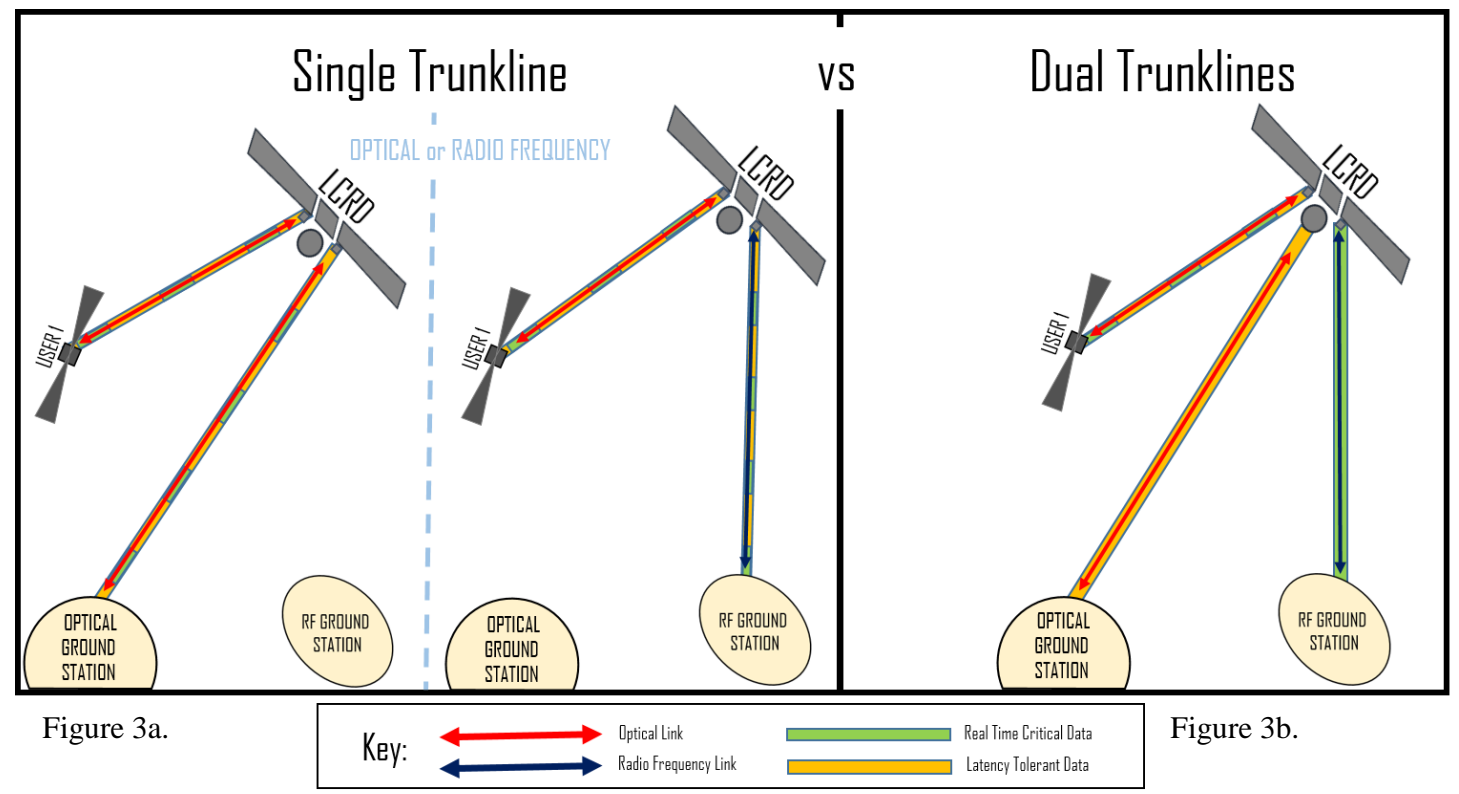

Figure 3. Single vs Dual Trunkline

\section{B. Storage and Retransmission}

In the discussion so far, the assumption was made that the user spacecraft system would be responsible for the retransmission of any data lost due to optical trunkline outages; therefore, the user spacecraft must store all data until delivery is confirmed. If a relay payload included the necessary channel encoders, channel decoders, frame processing, and storage, this responsibility for storage and retransmission can be moved from

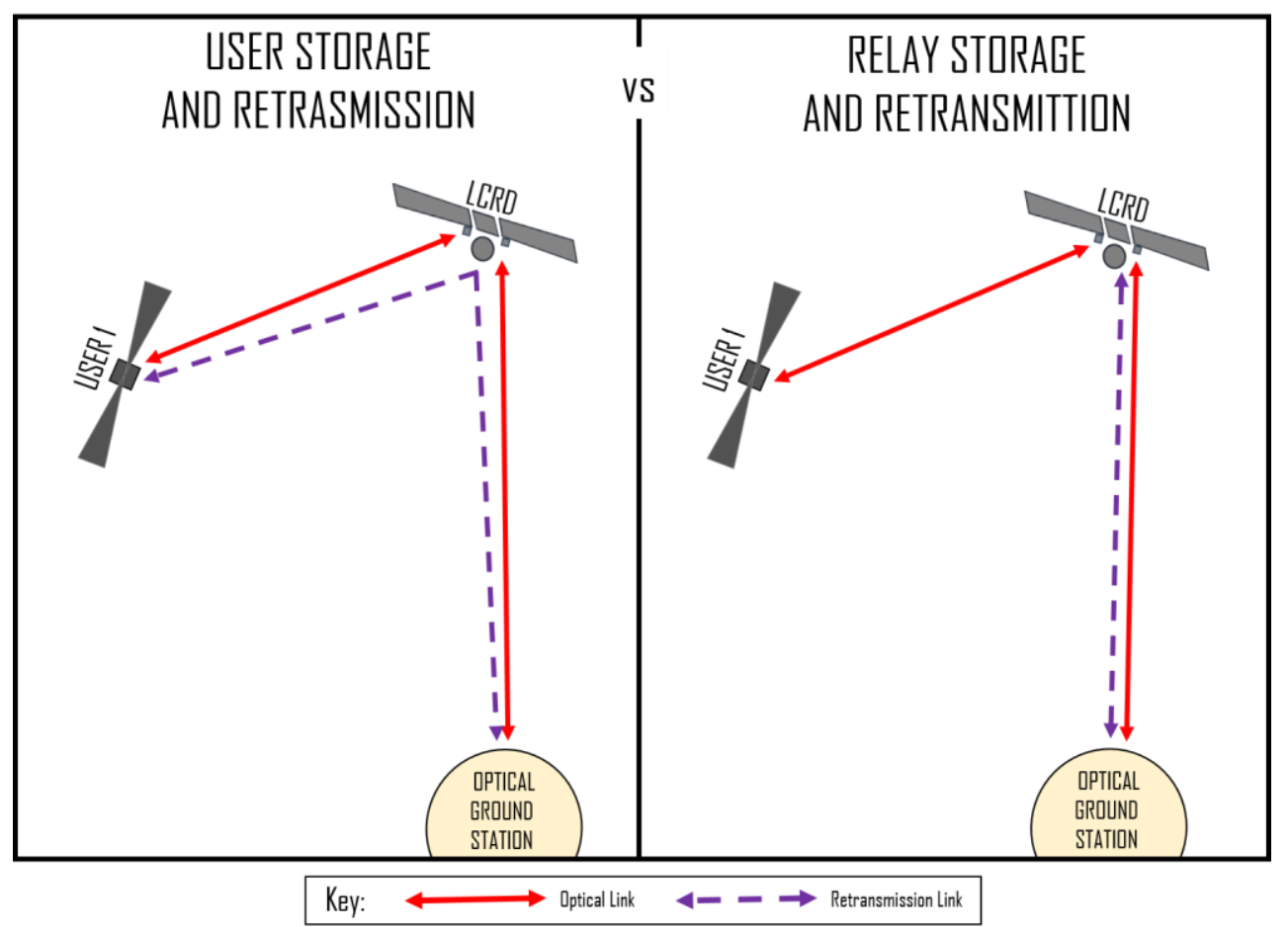

Figure 4. User vs Relay Storage and Transmission 
the user spacecraft to the relay. [Fig 4]. This is a significant increase of complexity for the relay payload, but provides several new efficiencies for the user spacecraft. This onboard capability will allow the trunkline to be packed even more efficiently, and provide a means for rate buffering and rate matching between available trunkline bandwidth and user link data rates. This approach also allows the decoupling of the user optical link channel coding and link protocols from those used for the trunklines or any other next hop. For example, space-to-space user optical links would not need to incorporate coding or interleaving to accommodate atmospheric effects, since the relay would perform any functions necessary before forwarding the data.

The Delay/Disruption Tolerant Networking (DTN) protocols provide a standard means for this network layer service. This has been demonstrated multiple times, including over lasercom links [7], and used operationally on the ISS [8]. The DTN protocols can be implemented on user spacecraft and the relay ground station independent of the relay payload; providing the storage management and retransmission protocols required for operation with inevitable optical link outages. The core DTN protocol, Bundle Protocol (BP), allows for standard networked communications in a store-and-forward manner [9].

\section{Relay Muxing and Switching}

The previous trades have discussed return link data flows (data originating on the user spacecraft and relayed to Earth), but, with a relay DTN node, analogous benefits can also be seen in forward link traffic (data originating on the Earth and relayed to the spacecraft). Critical realtime forward link traffic could be carried up the RF trunkline and muxed on the relay with other data received at the relay from the optical trunkline. Muxing and switching on the relay also allows for ops concepts in which data can be relayed with another spacecraft while each spacecraft also communicates with its earthbased operations center via the RF trunkline [Fig. 5].

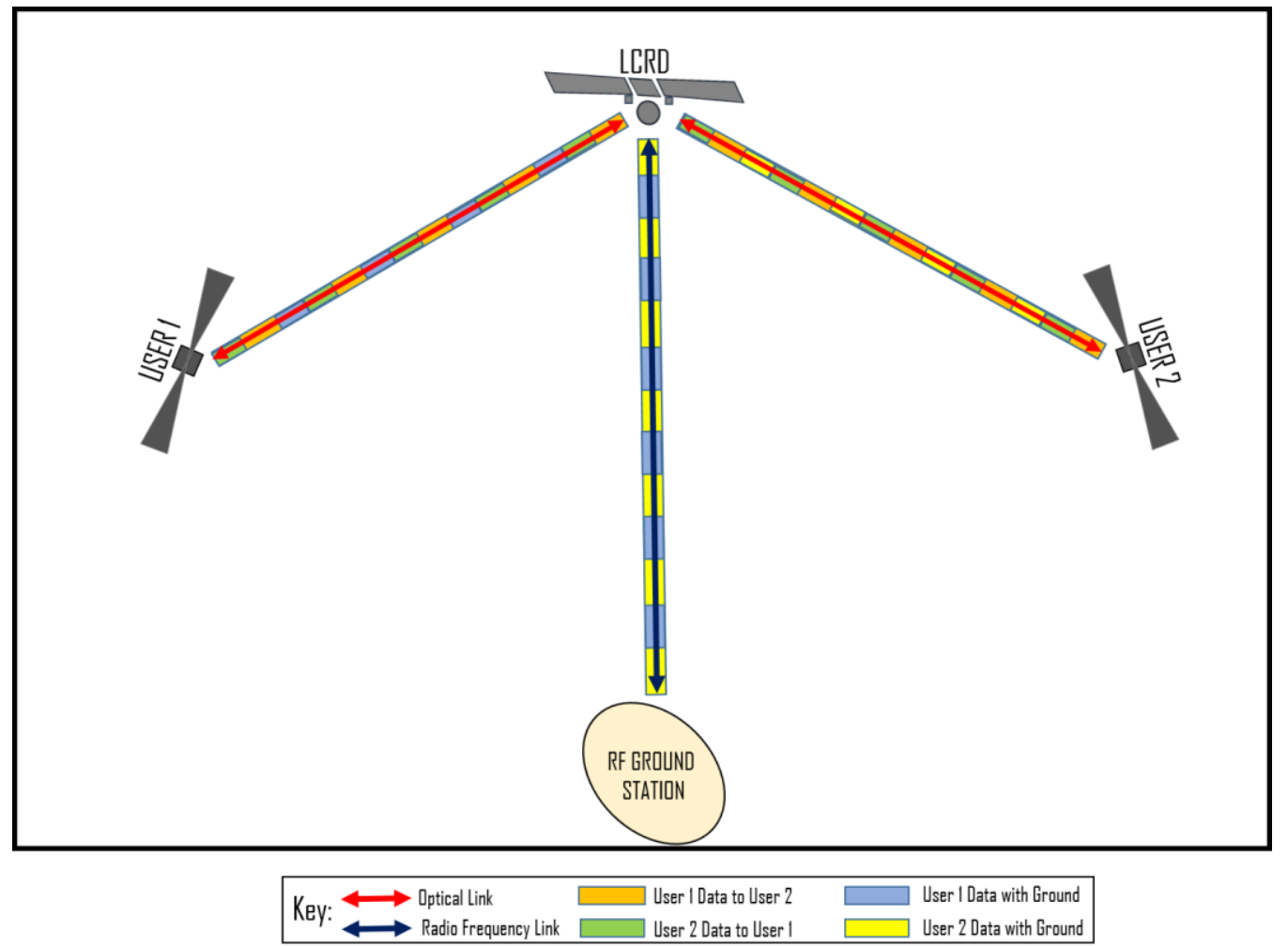

Figure 5. User to User Relay Muxed with Space-Ground Data 


\section{Other Trades}

Following these higher level trades, specifications for future relays will need to be developed for specific parameters. A combination of the available RF spectrum, maximum optical link rate, allowable optical link outage durations, costs of optical ground stations, and other factors will lead to a determination of the ratio of amount of trunkline bandwidth to be implemented between optical and RF. Further considerations of operations complexity, available relay SWaP, and costs will drive the trades that determine how much of the end-to-end system burden is placed on the relay instead of the users.

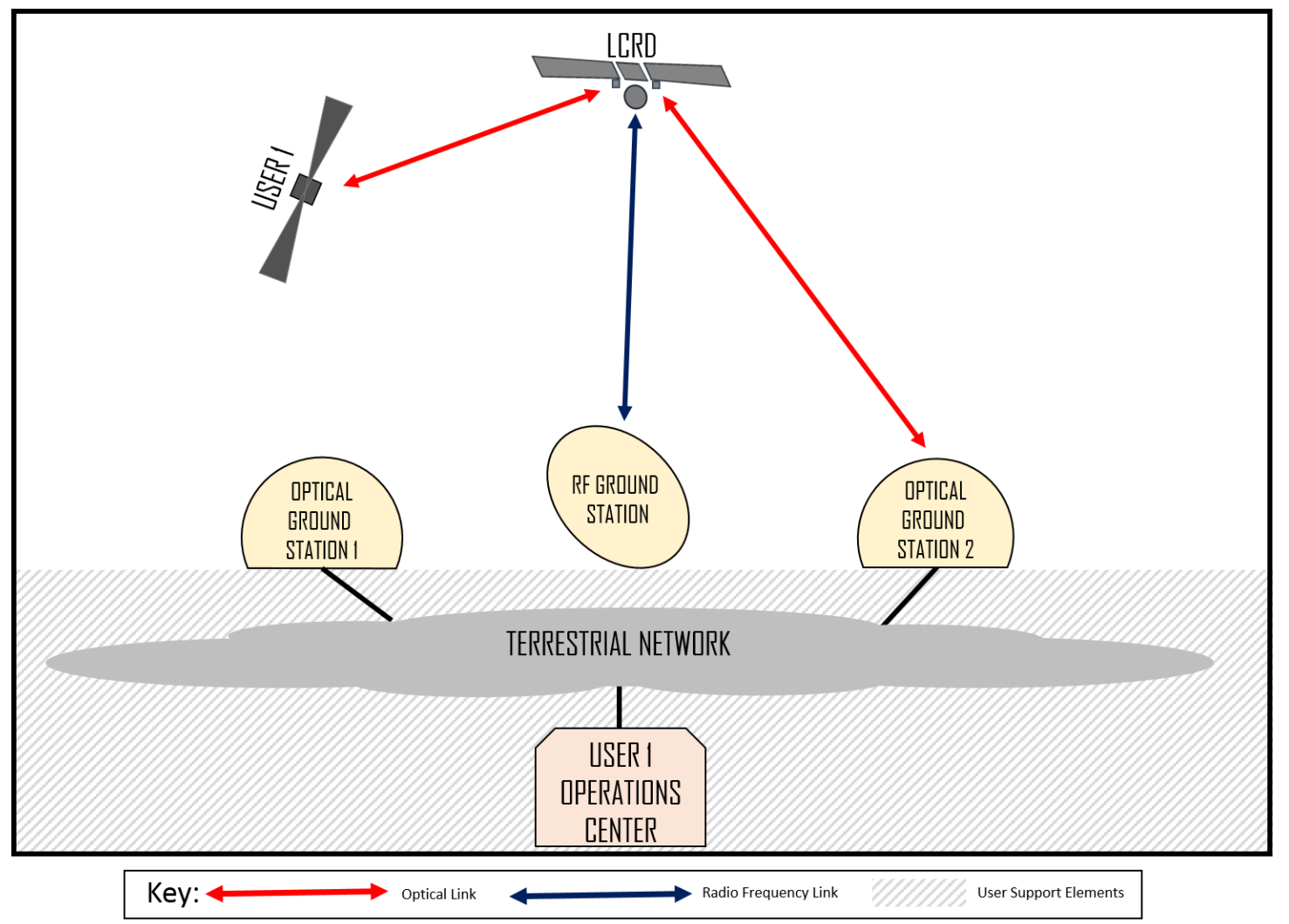

Figure 6. LCRD Supporting Orbiting User with Optical and RF Trunklines

\section{Conclusion}

During the two years of LCRD's on-orbit experiment phase, the concepts described in this paper that do not require changes to the LCRD flight payload will be demonstrated. These include all options demonstrating the single trunkline and the encoded frame link layer switch. As an operational demonstration mission, the system will be available to attempt different options to obtain experience in the implementation and operations challenges to inform the development of specifications for the future operational systems. LCRD will demonstrate how the combination of both RF and optical trunklines allows the full potential of optical communications user links to be realized, as the first users arrive during the post experiment phase. [Fig. 6]

\section{Acknowledgments}

LCRD is funded by the NASA Human Exploration and Operations (HEO) Mission Directorate's Space Communications and Navigation (SCaN) Program and the Space Technology Mission Directorate's Technology Demonstration Missions (TDM) Program. The author thanks the LCRD Project team for their diligent efforts to incorporate the RF trunkline into the flight payload. Lastly, the author would like to acknowledge Carolyn Crichton, whose editing and graphics support helped to make this paper possible. 


\section{References}

[1] Israel, David J., Bernard L. Edwards, Keith E. Wilson, and J. D. Moore. "An optical communication pathfinder for the next generation tracking and data relay satellite." In SpaceOps 2014 Conference, Pasadena, CA, USA, pp. 5-9. 2014.

[2] Boroson, Don M., Bryan S. Robinson, Curt M. Schieler, Farzana I. Khatri, Steven Constantine, Bryan M. Reid, and Donald M. Cornwell. "A New Optical Communication Architecture for Delivering Extremely Large Volumes of Data from Space to Ground", AIAA SPACE 2015 Conference and Exposition, SPACE Conferences and Exposition, (AIAA 2015-4658)

[3] Hauschildt, Harald, Francois Garat, Heli Greus, Khalil Kably, Jean-Pascal Lejault, Hermann Ludwig, Andrew Murrell Moeller et al. "European Data Relay System-one year to go!." Proc. ICSOS (2014): S1-3.

[4] European Space Agency "Space Data Highway: first laser transmission of an image taken by the Sentinel 1A satellite", URL: http://www.edrs-spacedatahighway.com/news/items/first-image [cited 22 August 2016].

[5] Edwards, Bernard, David Israel, Armen Caroglanian, James Spero, Tom Roberts, and John Moores. "A Day in the Life of the Laser Communications Relay Demonstration Project." In 14th International Conference on Space Operations, p. 2590.2016.

[6] Interagency Operations Advisory Group, “Optical Link Study Group Final Report,”IOAG.T.OLSG.2012. 5 June 2012 [7] Israel, David J., Donald M. Cornwell, Gregory D. Menke, W. John Guineau, "Demonstration of Disruption Tolerant Networking across Lunar Optical Communications Links." AIAA Space 2014, San Diego, CA, 2014.

[8] National Aeronautics and Space Administration "New Solar System Internet Technology Debuts on the International Space Station", URL: https://www.nasa.gov/feature/new-solar-system-internet-technology-debuts-on-the-internationalspace-station [cited 22 August 2016]

[9] Scott, K. and S. Burleigh, "Bundle Protocol Specification", RFC 5050, November 2007 